\title{
Summarizing Remarks
}

\author{
R. A. Marcus \\ Noyes Laboratory of Chemical Physics, $\dagger$ California Institute of Technology, \\ Pasadena, CA 91125, USA
}

\section{Introduction}

This Discussion has been exciting and broad in scope. We are particularly indebted to Professor John Simons and his organizing committee for having arranged this interestingly varied meeting.

Reflecting on how much the field has developed in the past half-century, I recall my first exposure, an indirect one, to a Faraday Society Discussion, 'The Labile Molecule' in 1947. E. W. R. Steacie, with whom I was doing post-doctoral research at the National Research Council of Canada, was about to go to England for the Discussion and showed me a paper of Smith and Taylor ${ }^{1}$ whose results were related to those ${ }^{2}$ he was going to present. This period saw the beginning of the measurement of the detailed kinetics of many free-radical reactions, and Smith and Taylor had generated methyl radicals from the photolysis of dimethyl mercury. ${ }^{1}$ In the presence of various hydrocarbons $\mathrm{RH}$, products such as $\mathrm{CH}_{4}$ and $\mathrm{C}_{2} \mathrm{H}_{6}$ were formed:

$$
\begin{aligned}
\mathrm{Hg}\left(\mathrm{CH}_{3}\right)_{2} & \stackrel{h \nu}{\longrightarrow} \mathrm{Hg}+2 \mathrm{CH}_{3} \\
\mathrm{CH}_{3}+\mathrm{RH} & \stackrel{k_{2}}{\longrightarrow} \mathrm{CH}_{4}+\mathrm{R} \\
\mathrm{R}+\mathrm{CH}_{3} & \stackrel{k_{3}}{\longrightarrow} \mathrm{RCH}_{3} \\
2 \mathrm{CH}_{3} & \stackrel{k_{4}}{\longrightarrow} \mathrm{C}_{2} \mathrm{H}_{6}
\end{aligned}
$$

together with many other steps, ${ }^{1}$ not shown. Using even only steps (1)-(4), a fairly complicated steady-state algebraic expression was obtained for the $\mathrm{CH}_{3}$ concentration and, from it, an expression for $R_{\mathrm{CH}_{4}}$, the rate of methane formation. ${ }^{1,2}$ The expression may ${ }^{1}$ or may not ${ }^{2}$ have been adequate for their purpose, but as I noted to Steacie one could use, instead, the simple relation

$$
R_{\mathrm{CH}_{4}} / R_{\mathrm{C}_{2} \mathrm{H}_{6}}^{1 / 2}=k_{2}[\mathrm{RH}] / k_{4}^{1 / 2}
$$

regardless of the complexity of the mechanism, an expression later used in our 1948 paper. ${ }^{3}$ For a series of RHs the relative rate constants $k_{2}$ of reaction (2) could then be determined. Steacie showed more excitement over that suggestion, and later mentioned that he gave it at the Discussion (not recorded, however), than he did, I thought, over some of my experiments. In any event, when compared with the extremely high level of theoretical results presented by experimentalists in the current Discussion the incident gives some hint at how much the field has developed during these past four to five decades!

\section{Definitions: 'Transition States' and Transition State}

The present Discussion has been concerned with transition states in two distinct senses of the term.

\footnotetext{
$\dagger$ Contribution no. 8481 .
} 


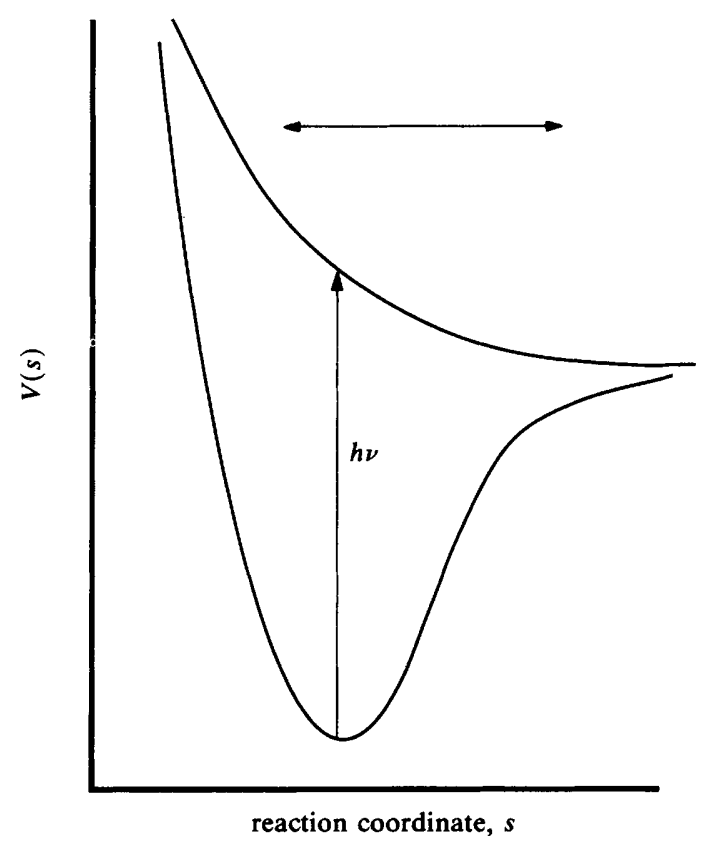

Fig. 1 Plot of a profile of two potential-energy surfaces for a photoexcitation versus a reaction coordinate $s$ and showing the 'transition states' (double-tipped arrow)

1. 'Transition states,' or transition region, denoting any configurations between those of reactants and those of products. ${ }^{4}$

2. The transition state, as defined in kinetics, denoting an optimal $2 N-1$ dimensional surface in a $2 N$ dimensional phase space, namely the surface for which there are the fewest (or no!) recrossings by trajectories proceeding from reactants to products and by trajectories from products to reactants. ${ }^{5}(N$ is the number of coordinates per reacting molecule in a unimolecular reaction or per reacting pair in a bimolecular one.)

Wigner pointed out that when there are no recrossings, and when a statistical equilibrium exists for the reactants, transition-state (TS) theory becomes exact in this classical description. ${ }^{5}$ He had replaced, thereby, the usual ad hoc quasi-equilibrium assumption in transition-state theory by an argument fully justified, instead, by classical dynamics and statistical mechanics. An example of 'transition states' is given by the double-tipped arrows in Fig. 1 and 2, while an example of the transition state is given by the dagger in Fig. 2 and 3. In some bimolecular associations or unimolecular dissociations there is no pronounced saddle-point in the potential-energy surface for the reaction coordinate (Fig. 3), and in that case the position of the transition state is determined variationally, ${ }^{6-8}$ so as to obtain the hypersurface with the fewest recrossings. ${ }^{5,6}$

A question that can be asked is whether each thermal reaction has an assignable transition state. Reactions for which there are complications are expected to be rare and include those systems for which the potential-energy surface contains a particular bifurcation. The latter, which can be termed a 'tilted monkey seat on the side of a hill,' is described in the Bernstein Memorial issue of the Journal of Physical Chemistry. ${ }^{9}$

In these summarizing remarks we address several aspects: the observations themselves, the question of what developments here are new for a Faraday Discussion, the extent to which some of these data extend bridges to other fields, some absentee but 


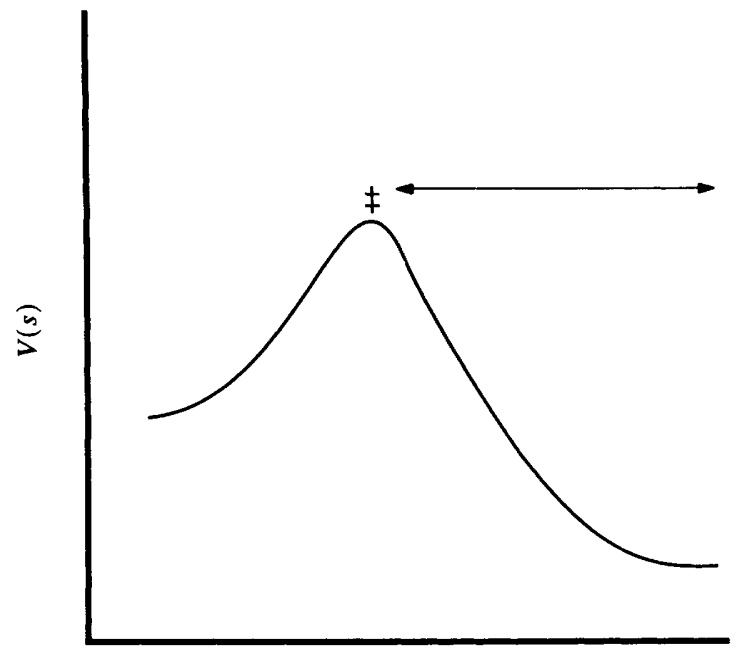

reaction coordinate, $s$

Fig. 2 Plot of a profile of potential-energy surface versus the reaction coordinate $s$ for a reaction containing a saddle-point, the 'transition states' region is shown with a double-tipped arrow. The transition state is indicated by $\ddagger$

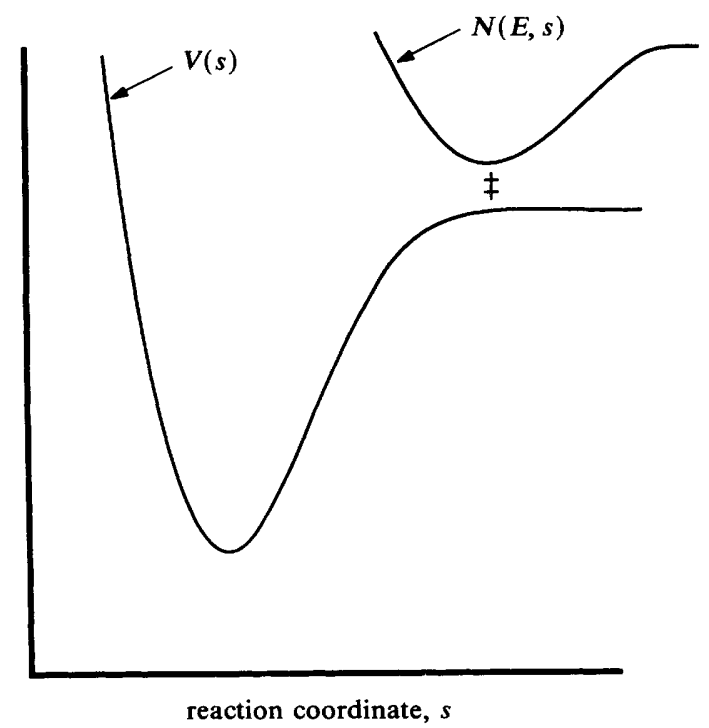

Fig. 3 Plot of a profile of a potential-energy surface versus the reaction coordinate $s$ for a reaction showing no saddle-point (no hump and the exit channel). The transition state is indicated by $\ddagger$

relevant topics not treated in the Discussion, several theoretical concepts and whether or not there is lurking in the field some unifying theory, yet to be found.

\section{Experimental Data}

We have seen in this Discussion many new experimental results and techniques in which in some cases the transition state itself is studied, and in others the 'transition states,' as defined above: 


\section{The Transition State}

(a) Direct measurements of the transition state described here include the timeresolved study using femtosecond excitation $\left(\mathrm{HgI}_{2}\right),{ }^{10}$ and spectroscopic measurements involving photoelectron detachment ( $\mathrm{XHY}^{-}$, with $\mathrm{X}$ and $\mathrm{Y}$ being various halogens, and $\left.\mathrm{FH}_{2}^{-}\right)^{11}$ and photoexcitation $(\mathrm{CaHCl}) .^{12}$

(b) Indirect studies of the transition state include measurements of rate constants as a function of temperature or, in unimolecular processes, energy and/or temperature, ${ }^{13}$ reaction cross-sections, ${ }^{14}$ differential scattering cross-sections $\mathrm{d} \sigma\left(\boldsymbol{k}, \boldsymbol{k}^{\prime}\right) / \mathrm{d} \Omega$ ( $\boldsymbol{k}$ and $\boldsymbol{k}^{\prime}$ are pre- and post-collision velocities), the rotational $j^{\prime}$, vibrational $v^{\prime}$, translational distribution of the reaction products, ${ }^{15,16}$ state-tostate cross-sections $\mathrm{d} \sigma\left(\boldsymbol{k}, v, j, \boldsymbol{k}^{\prime}, v^{\prime}, j^{\prime}\right) / \mathrm{d} \Omega,{ }^{17}$ and various vector correlations. ${ }^{18,19}$ Related quantities or concepts are described for reactions on surfaces. ${ }^{20-22}$

\section{2. 'Transition States'}

(a) Direct information on 'transition states' is obtained from time-resolved (e.g. $\mathrm{NaX}, \mathrm{ICN}, \mathrm{CH}_{3} \mathrm{I}, \mathrm{X}-\mathrm{HOCO}$ reactions) ${ }^{10}$ and spectroscopic (e.g. photodissociation spectra of $\mathrm{HONO}, \mathrm{HCO}_{2} \mathrm{H}$ and $\left.\mathrm{CH}_{3} \mathrm{ONO}\right)^{23,24}$ measurements.

(b) Indirect information on this region is obtained from the measurements of the rotational-vibrational-translational distribution of the reaction products. ${ }^{24-26}$

In addition to the experimental results above, a number of the papers have been primarily concerned with various theoretical aspects, ${ }^{27-32}$ including the resonances in heavy-light-heavy (e.g. I $+\mathrm{HI})$ chemical reactions, ${ }^{27}$ transition-state spectroscopy for $\mathrm{NaI}, \mathrm{O}_{3}$ and $\mathrm{FHF}^{-}$dissociations, ${ }^{28}$ wavepacket quantum calculations for $\mathrm{NaI}$ and $\mathrm{CH}_{3} \mathrm{ONO},{ }^{29}$ laser catalysis of the $\mathrm{H}+\mathrm{H}_{2}$ reaction, ${ }^{30}$ cumulative reaction probability for the $\mathrm{H}+\mathrm{H}_{2}$ reaction, ${ }^{31}$ and the photodissociation of $\mathrm{CH}_{3} \mathrm{I}$ under short laser pulses, including non-adiabatic effects. ${ }^{32}$

The main focus of the present Discussion being the transition state, the position of the transition state $s^{\ddagger}$ along a reaction coordinate $s$ is clearly of particular interest. It is useful to review first several existing forms of the TS theory of reaction rates. In standard TS theory, $s^{\ddagger}$ is chosen to occur at the saddle-point, if any, of the potentialenergy surface.

In variational transition-state theory ${ }^{6-8} s^{\ddagger}$ need not occur at the saddle-point, even when it exists, and is dependent on the energy $E$ (microcanonical variational TS theory) and on the total angular momentum $J$ or on the temperature $T$ (canonical variational TS theory) and $J$. Originally, the treatment of all coordinates in variational TS theory was classical. ${ }^{6}$ In a now common version of microcanonical transition-state theory, the motion along the reaction coordinate $s$ is treated classically and all other coordinates are treated quantum mechanically, i.e. their properties are calculated at each fixed $s$. At a given $E$ and $J$ and at a given value of the reaction coordinate $s$, the flux of systems from the reactants' region to the products' region, is proportional to the number of vibrational-rotational states $N(E, J, s)$ of the system at that $E, J$ and $s$. Minimizing $N(E, J, s)$ with respect to $s^{7,8}$ provides the value $s^{\ddagger}$ of $s$ where the flux is least and hence where there is the least recrossing (Fig. 3). This $s^{\ddagger}$ serves thereby as the best choice for the transition state, for the given choice of reaction coordinate $s$. One can modify this formulation by replacing the classical description of the $s$ motion by one containing some appropriate nuclear tunnelling factor.

In vibrationally adiabatic transition-state theory (a term introduced in $1965^{33}$ but based on ideas which originated much earlier ${ }^{34}$ ), $s^{\ddagger}$ depends on the quantum state of the motion in the TS transverse to $s$ : the transition state in vibrationally adiabatic TS theory occurs at the maximum of the vibrationally adiabatic effective potential-energy curve for that quantum state, plotted $v s$. $s$. This state-dependent $s^{\ddagger}$ can be quite displaced from the saddle-point. 
Because of this variability in the position of $s^{\ddagger}$, a quantity defined for chemical-kinetic purposes rather than for most of the present observations, the experimental methods, direct or indirect as before, could be classified instead according to whether they provide information on the saddle-point region when it exists, or on the other regions of the potential-energy surface between reactants and products, or on both.

\section{Results New for a Faraday Discussion}

This Discussion contains a number of results of a type that do not appear to have been described previously at a Faraday Discussion, including:

1. Real-time observation of the transition state $\left(\mathrm{HgI}_{2}\right)$ and of the collision complex $\mathrm{Br}+\mathrm{I}_{2} ;^{10}$

2. Spectroscopic observation of the transition state $\left(\mathrm{FH}_{2}^{-}, \mathrm{Ca}^{*} \mathrm{HCl}, \mathrm{XHY}^{-}\right) ;^{11,12}$

3. Four-vector correlation of initial and final velocities and rotational angular momenta; ${ }^{19}$

4. Reactions of velocity-aligned atoms; ${ }^{14,18}$

5. Photoinduced charge transfer at surfaces; $;^{35}$

6. Use of periodic orbits in the interpretation of the structure in photodissociation spectra; ${ }^{23}$

7. Dynamics of surface reactions and the analysis using a potential-energy surface and concepts analogous to those used for gas-phase reactions. ${ }^{20-22}$

\section{Relation to other Fields}

A variety of connections exist between the research in this Discussion and that in other fields, an aspect which broadens its scope and, to others, its relevance and interest:

\section{Charge Transfer at Surfaces}

The photoinduced charge transfer at a metal electrode followed by bond rupture ${ }^{35}$

$$
\mathrm{Ag}^{(-)}(111) / \mathrm{CCl}_{4} \stackrel{h \nu}{\longrightarrow} \mathrm{Ag}(111) / \mathrm{CCl}_{3}+\mathrm{Cl}^{-}
$$

is related to well known electrochemical reactions in solution such as

$$
\mathrm{M}^{(-)} / \mathrm{RCl} \longrightarrow \mathrm{M} / \mathrm{R}+\mathrm{Cl}^{-}(\text {soln })
$$

where $\mathbf{M}$ is a metal electrode and $\mathbf{R}$ a free radical, reaction (7) being followed by other reactions of $R$. The study of reactions involving some organic bridge $B$ suitably bound to the electrode $M$,

$$
\begin{gathered}
\mathrm{M}^{-} / \mathrm{BCl} \stackrel{h \nu}{\longrightarrow} \mathrm{M} / \mathrm{B}+\mathrm{Cl}^{-} \\
\mathrm{M}^{-} / \mathrm{BOx} \stackrel{h \nu}{\longrightarrow} \mathrm{M} / \mathrm{B} \operatorname{Red}
\end{gathered}
$$

as a function of the length and nature of the bridge $B$ would be of particular interest. Here, $\mathrm{Ox}$ is the oxidized form of an outer-sphere electron-transfer reactant and Red contains the transferred electron. Such studies would provide an interesting comparison with other investigations ${ }^{36,37}$ of bridged electron transfers in condensed phases, e.g. cited and treated in ref. 37 . We have also commented on this aspect elsewhere in this Discussion. 


\section{Intramolecular Electron Transfer in the Gas Phase}

Intramolecular electron transfers have been studied in the gas phase and in solution (though not at this Discussion). A reaction examined in the gas phase ${ }^{38}$ that is a prototype of one much studied in solution ${ }^{39}$ is<smiles>CN(C)c1ccc(S)cc1</smiles>

where the $n \mathrm{~S}$ denote polar solvent molecules which can stabilize the charge separation.

\section{Inorganic Reactions}

A number of reactions treated are related to inorganic reactions such as 'hydrogen activation,' an example in the present Discussion being ${ }^{20}$

$$
\mathrm{Cu}(110) / \mathrm{H}_{2} \longrightarrow \mathrm{Cu}(110) / \mathrm{H}
$$

where $\mathrm{H}_{2}$ is in a $v=1$ vibrational state ${ }^{20}$ and where only one of the metal-bound $\mathrm{Hs}$ in the products is shown. Another reaction studied here is related to the embrittlement of metals. $^{22}$

\section{Interstellar Chemistry}

This subject, absent here but a natural area for application of current techniques used in this Discussion, is the subject of a Faraday Symposium in 1992.

\section{Absent Friends}

It is not practical in a short symposium such as the present one to address all topics pertinent to transition states, and several topics which are absent here and for which we bow our heads in a momentary silence are:

1. Raman studies of 'transition states';

2. Unimolecular reactions, treated in a Faraday Discussion in 1983. (They too, have transition states!) Indeed, the broad field of reaction rates themselves, which could occupy many Discussions, was intentionally made absent;

3. Ion-molecule reactions, treated in a Faraday Discussion in 1987;

4. Clusters, treated in a Faraday Symposium in 1989.

\section{Concepts and Theories}

Various questions that arise in a field are, we recognize, usually phrased in the context of a comparison with some theory or theoretical concept. Before considering several questions which arose during the Discussion, we list here several concepts or theories that have been introduced into the present field.

1. Theories such as collision theory, transition-state theory, RRKM theory, phasespace theory, the statistical adiabatic channel model, semiclassical theory (manydimensional WKB) and the infinite-order sudden approximation, among others.

2. Use of the well known skewed-axes diagram ${ }^{41}$ for describing collinear reactions, which brings out rather vividly the dynamical constraint imposed by conservation 
of linear momentum. Concepts such as early or late barrier in a reaction ${ }^{42}$ and the nature (vibrational vs. translational) of the attendant energy release, known to the founding fathers (e.g. ref. 41), received a new lease, elaboration and tests using modern computers. ${ }^{4}$

3. Classical trajectory methods, involving numerical solutions of Newton's classical equations of motion.

4. Numerically obtained quantum-mechanical solutions of the Schrödinger equation, ${ }^{27,28,43,44}$ including some in this Discussion using hyperspherical coordinates. ${ }^{27,28}$

5. Dynamical treatments in which low-frequency coordinates are treated classically and the high-frequency ones quantum-mechanically.

6. Various wavepacket-inspired concepts and treatments.

7. Specific dynamical concepts such as vibrational adiabaticity for some reactions, ${ }^{33,34}$ which in turn is based, in practical terms, on introducing a continuously adjusting set of coordinates, natural collision coordinates, along a reaction path. ${ }^{45}$ These coordinates and Hamiltonian now also appear frequently, after extension to more coordinates and omission of anharmonic and Coriolis (rotationalinternal coordinate) terms, as the "reaction-path Hamiltonian. ${ }^{46}$

8. A statistical theory for reaction rates (e.g. variational RRKM) supplemented by an assumption for dynamics in the exit channel so as to permit the prediction of the quantum-state distribution of the products. ${ }^{47}$ E.g. for this purpose, for unimolecular dissociations (as in Fig. 3) having no marked potential-energy hump in the exit channel, an assumption has been added to RRKM theory: It was assumed that the vibrations are adiabatic and the hindered and other rotations non-adiabatic, subject to total angular momentum conservation, during the motion along the reaction coordinate in the exit channel from the inner TS to a phase-space theory TS. $^{47}$

9. Use of periodic orbits and their neighbouring trajectories ${ }^{23,48}$ (a quantummechanical wavefunction does not correspond semiclassically to a single classical periodic orbit but rather, instead, to a 'more space-filling' trajectory or set of trajectories) to treat spectroscopic structural information and also to define a transition state for a reaction in a coordinate-free manner. In practice, these periodic orbits have been employed for this purpose only for a very small number of coordinates. To this aspect we may add concepts such as bifurcation of trajectories (unstable periodic orbits) and, in the limit, chaos.

10. Of particular importance to chemical dynamics, ideas related to the ab initio calculation of potential-energy surfaces.

\section{Questions}

Several random examples of questions that have been (or could be) raised at this Discussion, each theory-based, are the following.

1. How are the predicted resonances best observed in bimolecular reactions?

2. Is the spectroscopic structure in the photodissociation continuum better described in terms of Franck-Condon factors or resonances?

3. Is the structure in these photodissociation spectra well described in terms of periodic trajectories (and their neighbouring set of trajectories) ${ }^{23,48}$

4. Can one detect the stepwise increase in microcanonical rate constant with increasing energy in bimolecular ${ }^{31}$ or unimolecular ${ }^{49}$ reactions or in the yield of the product's rotational-vibrational quantum states, expected from TS theory? ${ }^{31}$

5. In a reaction such as

$$
\mathrm{CH}_{2} \mathrm{CO} \longrightarrow \mathrm{CH}_{2}+\mathrm{CO}
$$




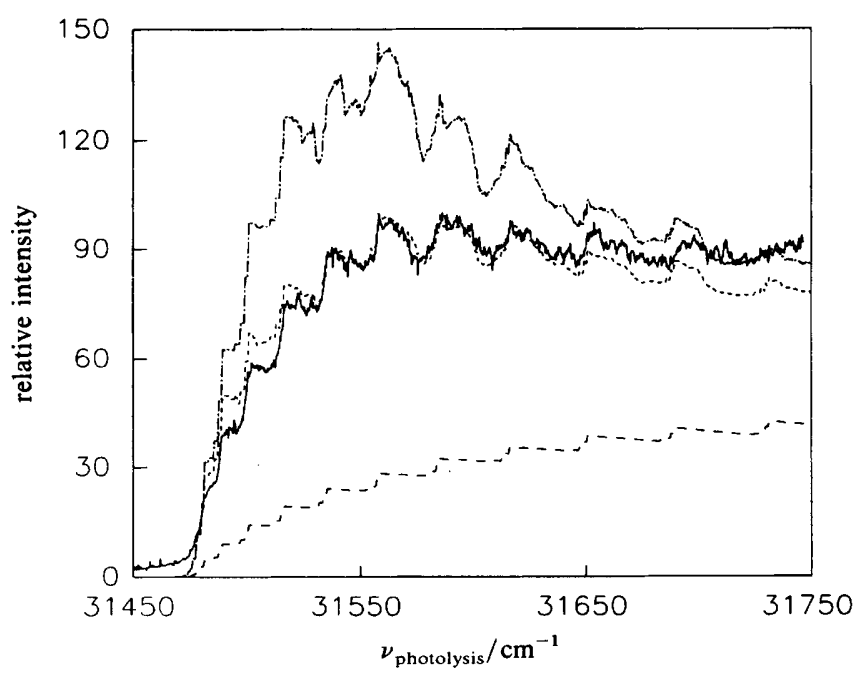

Fig. 4 Plot of photofragmentation excitation spectrum for $\mathrm{CH}_{2} \mathrm{CO}$ versus energy. ${ }^{50,51}$ The solid line is the experimental curve and the dashed or dotted lines various theoretical curves. The curve closest to the experimental one is that obtained in ref. 51

an analysis of $N(E)$ vs. $E$ yielded, in a narrow energy region $\left(c a .150 \mathrm{~cm}^{-1}\right)$ just above each vibrational excitation threshold, two simultaneously contributing transition states, a loose phase-space theory TS and, at a smaller separation distance, a tighter but still fairly loose TS. ${ }^{50}$ Can one observe in real time perhaps an oscillation or two between the two TSs in this energy interval?

6. What is the nature of the complementarity between the current time-resolved and spectroscopic observations for short-lived species $?^{12,29}$

7. What are the relative merits of hyperspherical coordinates and natural collision coordinates, and their Hamiltonians, for understanding TS theory and for treating the full reaction dynamics numerically?

8. Various rather specific questions arose, such as is there a positive correlation between rotational $j^{\prime}$ and vibrational $v^{\prime}$ state of the reaction products of $\mathrm{H}+$ $\mathrm{HR} \rightarrow \mathrm{H}_{2}+\mathrm{R}$ ? $^{16}$

We comment here on only some of these questions, including 2, 4, 6 and 7 . The results of quantum-mechanical calculations reported for rates and for yields for some bimolecular reactions in this Discussion illustrate the staircase behaviour in microcanonical rate constants referred to in question 4 . Since a microcanonical study for bimolecular rate constants or yields is not practical experimentally, however, such behaviour is best examined experimentally in unimolecular reactions, when there is a statistical intramolecular energy redistribution prior to reaction. An example, comparing experiment with variational RRKM theory and utilizing a dynamical assumption ${ }^{47}$ mentioned earlier for the exit channel, is given in Fig. 4 for the yield of ${ }^{1} \mathrm{CH}_{2}$ in reaction (11) versus energy. ${ }^{50,51}$ There was an increase in yield when each successive rotational state of $\mathrm{CO}$ became energetically accessible.

Regarding the complementarity in question 6, the time-resolved and the spectroscopic approaches have been used thus far to study the transition states of quite different reactions. ${ }^{10-12}$ For stable or fairly stable systems there is an additional type of complementarity: the spectroscopic data can yield numerous resolved lines, for example in the two-photon Doppler-free spectrum of benzene, ${ }^{52}$ which provides revealing information on Coriolis/anharmonic couplings. ${ }^{53}$ On the other hand, spectroscopic studies may 
also yield such a plethora of lines that in some cases it may be difficult to see an overall pattern. In this case either low-resolution spectra $^{54}$ or a coarse-graining ${ }^{55}$ of a well resolved spectrum have been useful. In the time-resolved spectrum, on the other hand, the resolution or the coaise graining is controlled by the time of the phenomenon itself or the observation time, hence separating the different timescales. ${ }^{10} \mathrm{~A}$ limited time for the probe measurement after the initial pulse avoids long-time recurrences, which, after a Fourier transformation, would correspond to small spectral splittings and so to a multiplication of the number of spectral lines. On a different aspect, lifetimes of short-lived species have been measured directly in time-resolved measurements and, via line broadening, indirectly in frequency-resolved experiments. The second of these methods can lead to spurious lifetimes (too short) because of alternative sources for the broadening.

In regard to question 7 , we note first that the use of a natural collision coordinatebased (reaction-path) Hamiltonian ${ }^{45,46}$ requires particular care (not always employed), because of the singularity of the coefficient of the kinetic energy $p_{\mathrm{s}}^{2}$ term when the path curvature is large. Perhaps for this reason, hyperspherical coordinates, ${ }^{56-58}$ whose singularity occurs much further from the reaction path, are more frequently used in numerical coordinate-based computations, e.g. in this Discussion, ${ }^{27,28}$ though the NRC Hamiltonian is also employed (e.g. ref. 43). In using hyperspherical coordinates for numerical calculations one appears to pay a price (larger basis set) but avoids the difficulty due to the singularity. The hyperspherical coordinates have been useful, too, for visualizing reaction kinetic and some dynamical properties of light-atom transfer reactions. ${ }^{59}$

For visualizing the basis of vibrationally adiabatic TS theory the natural collision coordinate system and an approximate Hamiltonian based on them is preferable. When it is used instead to treat the full chemical dynamics, the rotational excitation of the products for example, it is essential, ${ }^{43}$ of course, to include the Coriolis and anharmonic terms $:^{45}$ the motion of the separating fragments in a reaction is usua:ly best discussed in the TS region using a body-fixed frame, as in ref. 43 and 44 , but at larger separation distances the motion of the fragments is no longer constrained by that frame and, as is well known, is better treated in the laboratory-fixed frame. Coriolis terms also cause deviations from the statistical-adiabatic channel model (SACM). ${ }^{60}$

The rotational-vibrational-translational distribution of the products is, as noted earlier, a property dependent both on the transition state and on the 'transition states.' In the case of a reaction which has a transition state one distribution of interest is that in the TS itself. As the system then moves along the reaction coordinate from the TS to the products' region, this distribution is modified by any exit-channel effects. Earlier, we have noted an example of a simple dynamical assumption for the latter for unimolecular reactions when there is no hump in the potential-energy curve along the reaction coordinate in the exit channel. ${ }^{47}$ In the case of a photodissociation involving photoexcitation to a repulsive electronic state, Franck-Condon factors control the distribution among quantum states immediately after the excitation, a distribution which is then modified by subsequent exit-channel effects as the system moves along the repulsive surface to the products' region. This time-dependent description of a system moving either from a TS or along a repulsive surface after photoexcitation could, in an alternative theoretical formulation, be replaced by a time-independent description. When the experimental observation is of a short-time nature, the motion can be described using a wavepacket of these time-independent solutions.

Franck-Condon factors have been mentioned above. Their magnitude depends on the zeroth-order modes used to describe the wavefunctions in the initial and final electronic states. In the initial electronic state, usually having a low vibrational energy, a normal-mode description of the motion is customarily used, while in the final electronic state, where the vibrational energy is frequently high, the zeroth-order descriptions are 
usually based on normal modes, local modes (e.g. for $\mathrm{CHs}$ ), or local/normal modes. However, quantum-mechanical calculations and classical mechanical periodic orbit calculations have both demonstrated that an improved zeroth-order description of the vibrational motion in this high vibrational energy regime may be quite different from that presented by those simple modes. The new zeroth-order modes of motion, which are similar in shape to that of some periodic trajectories at that energy, have provided a new insight into the low-resolution spectra of those molecules. ${ }^{23,48}$ They are also typically unstable, being coupled to other modes and so giving rise to a resonance-like behaviour. When such a zeroth-order mode provides a reasonable description of the motion, but is coupled to a number of other modes, the low-resolution spectrum may appear diffuse, though at sufficiently high resolution, at least in the case of sufficiently long-lived electronic states of the molecule, will exhibit instead numerous lines arising from the 'exact' quantum states of the molecule as a whole. If the number of coupled states is sufficiently small, even the low-resolution spectrum may, of course, appear sharp.

One might add here that the periodic trajectories of interest are really signatures of the nature of the surrounding tori, or partially broken tori in phase space, ${ }^{61}$ each torus in a regular (non-chaotic) regime corresponding semiclassically to a quantum state. ${ }^{62}$

\section{Unifying Theory in Chemical Dynamics}

In this array of new experiments and multifaceted theoretical concepts one may ask whether there is perhaps some unifying and simple-appearing theory that is both physically appealing and sound. Such a theory would be patently a boon to the interpretation and prediction of experiments. To place this question in a broader context, it is useful to consider first examples of closed-form or highly insightful, even if somewhat numerical, theories in physical chemistry in general.

Examples of such theories are the various mean-field descriptions in statistical mechanics, including those for gases (van der Waals), liquid crystals and magnetic systems (Curie points); the Debye-Hückel theory of interionic interactions and their effect on thermodynamic and kinetic properties; the Born theory of ion solvation; the Debye treatment of the relative permittivity, modified by Onsager to include the reaction field of dipolar systems; the Clausius-Mossotti equation relating the refractive index of solutions to molecular polarizability; the scaled-particle theory of hard-sphere liquids; the Debye theory of specific heats; the Boltzmann equation for the one-particle phase space distribution function for transport properties of molecules in gases and liquids and of electrons or holes in the solid state; the Bloch equation using $T_{1}$ and $T_{2}$ lifetimes to treat the dynamics of magnetic (spin) systems; the quantum-mechanical description of molecular rotational and vibrational quantum states (including those of 'Morse-like' molecules, as well as of harmonic oscillator ones) and the application to molecular spectra and to thermodynamic and kinetic properties; the free-electron and tight-binding models for electrons in solids; the Thomas-Fermi treatment of the electronic properties of atoms; the London formula for long-range intermolecular interactions due to dispersion forces; the quantum-defect expression for molecular Rydberg states; the molecular orbital theory for molecular spectra; the valence-bond theory for chemical bonding; the Woodward-Hoffmann molecular orbital rules for the role of orbital symmetry in thermal and photochemical reactions; the transition-state theory for reaction rates in general, with and without nuclear tunnelling; the theory of electron-transfer reactions in solutions, at electrode and other interfaces, and in photo-induced ET processes; the WeeksChandler-Andersen theory of liquids; the Kramers' treatment of frictional effects on reaction rates; and, although not a closed-form theory, the recently developed expression for nuclear tunnelling rates in thermal chemical reactions using Monte Carlo methods and the properties of Feynman 'centroids'; ${ }^{63}$ radiationless transition theory and its application to spectroscopic and reactive properties of few- or many-level systems of 
isolated molecules; the Bixton-Jortner model for these systems. Other significant developments involving, in part, numerical computation for implementation, include density-functional theories for molecules and solids, quantum Monte Carlo calculations of equilibrium ${ }^{64,65}$ and dynamical properties ${ }^{65}$ of thermally averaged systems, and the application of a flux-flux quantum expression ${ }^{66}$ in a Monte Carlo calculation of the rate constant.

We consider next what appears to be the situation in the field of chemical dynamics of largely state-to-state behaviour. While the subject of the present symposium is the transition state, the intention has been to focus on dynamical and spectroscopic aspects rather than on thermal rate constants, and indeed essentially all of the papers and discussion comments have the dynamical and spectroscopic aspects as their central themes. Treatment of thermal reaction rates, included in the above listing, are omitted in the following.

In chemical and molecular dynamics there are many closed-form or insightful theories for specific processes, and we first recall briefly some of them. One of the earliest was the Jackson-Mott treatment of translational-vibrational transition probabilities, another being the Landau-Zener treatment of curve-crossing phenomena. Other treatments include 'forced-oscillator' theories of translational-vibrational energy transfer collisions, used also for the behaviour in entrance or exit channels of chemical reactions; a power-law theory of Pritchard for rotational-translational collisional energy transfer; 'semiclassical' theories for collisions or other processes, in which one coordinate is treated classically and the remaining coordinates quantum-mechanically; 'sudden approximations' in molecular collisions; the Franck-Condon overlap treatment of the vibrational quantum-state distribution of the products of exothermic chemical reactions; phase-space, statistical adiabatic channel model and RRKM theories of energy and angular momentum-dependent unimolecular reaction rates, and of the rotational-vibrational quantum-state distribution of the reaction products, the RRKM theory having been recently extended to include the latter; the adaptation ('angular momentum + RRKM') by Herschbach and co-workers of statistical unimolecular theory to the formation of molecular complexes in crossed molecular beams and their unimolecular disappearance to form products; vibrationally adiabatic concepts and the use of the natural collision coordinate/reaction Hamiltonian approach to provide a basis for understanding when vibrational adiabaticity tends to occur in a reaction, and when it does not; sudden-switching models from entrance to exit channels in chemical reactions, for providing at least some qualitative insight into the vibrational-state distribution of the products of chemical reactions; and the use of semiclassical theory and/or ideas in non-linear mechanics (periodic orbits, role of chaos) either at the semiclassical or the purely classical level to treat some dynamical results mentioned later. This list is a rather long one, but is not intended to be exhaustive.

We see that for chemical dynamics there has been a plethora of theories, designed for individual classes of systems and for individual limiting situations in those systems. Perhaps this is the way it will be. However, in the field of physical chemistry of equilibrium properties in general, many topics of which were mentioned earlier, there has often been some common denominator, namely the molecular-based canonical partition function, or the dielectric or elastic continuum variants thereof. For kinetic properties of thermal systems, in the case of small deviations from equilibrium, the correlation function formalism of Kubo and others has played an analogous role. In the case of large or small deviations from equilibrium, with an added approximation, so has the Boltzmann equation. There has been considerable progress recently in such unified calculations (numerical), Monte Carlo-based, of equilibrium and kinetic properties using, respectively, partition function and correlation function formalisms. ${ }^{63-66}$

Experimental data on chemical dynamics, described at this symposium, are, however, much more detailed (e.g. 'state-to-state') than those obtained in thermally averaged 
experiments, and correspondingly, the demands on theory are of a more detailed nature. A bridge from the thermal to the state-to-state level is the microcanonical system. The experimental systems so treated are, in practice, unimolecularly reacting systems having a given vibrational energy and either a specified total angular momentum $J$ or some distribution of $J$ s corresponding to a particular rotational temperature $T$, a $T$ bearing no relation to the total vibrational energy. Monte Carlo-based ensemble techniques, used now for canonical ensembles, can, in principle at least, be adapted to such systems, though presumably with greater difficulty because of the oscillatory nature of the integrands for microcanonical systems.

For dynamical systems whose energy is not merely known but about which data are obtained at a more detailed state-to-state level, the basic theory at first glance might seem to be the Schrödinger equation or Newton's equations of motion. But for treating quantum-dynamical phenomena in reactions and in energy-transfer collision, it would be useful to have instead a theory based, of course, on the Schrödinger equation but containing approximations which make it immediately and relatively simply adaptable to the calculation of chemical-dynamical properties.

A theory which in principle has this kind of generality for chemical dynamics, rather than being specialized at the onset to specific classes of systems or for specific models, is semiclassical theory (generalized WKB theory). Semiclassical theory has played an important and stimulating role, and its influence is seen today in nuclear tunnelling, in periodic-orbit based concepts, and in the calculation of thermally averaged reaction rates and other thermally averaged properties. Originally, semiclassical theory ${ }^{67-70}$ (generalized WKB theory) seemed to be a good candidate for a unifying theory for state-to-state dynamics: the expressions for the semiclassical wavefunction or propagator $^{67}$ and for the experimental observables, such as the differential scattering crosssection, are simple in appearance and have a certain physical intuitiveness. ${ }^{68-70}$ They provide an insight into quantum effects, in particular nuclear tunnelling and interference. As Dick Bernstein mentioned to me many years ago, one of the virtues of semiclassical theory is to give a basis for a justification, and an understanding of the limitations, of the then (and now) widespread use of classical-mechanical trajectories for interpreting various experimental data in chemical dynamics.

To be sure, there were limitations to semiclassical theory: it was by no means a purely analytical approach since, at least as customarily used, numerically obtained trajectories were employed, sometimes analytically continued into the classically forbidden region, to calculate the experimental quantities. Nevertheless, the beauty of its overall structure lent an air of universality to it. However, apart from certain situations which typically involve few coordinates, quantum-mechanical rather than semiclassical methods are currently being used to treat, instead, real problems in chemical dynamics. The reasons include the frequently chaotic nature of many trajectories and the timeconsuming 'root search' needed to find the desired trajectories for a state-to-state stationary phase or uniform approximation (e.g. ref. 71 for a fully $3 \mathrm{D}$ calculation). An alternative integral expression, ${ }^{68-70}$ for the $S$-matrix elements, using classically allowed trajectories avoids the root search problem and has been used in a 3D rotationaltranslation energy-transfer calculation. ${ }^{72}$ Problems arose in the calculation of inelastic ${ }^{72}$ and reactive (unpublished) studies, in part due to bifurcating trajectories.

Examples of how semiclassical theory provides insight into tunnelling and interference processes and via optimal ${ }^{73}$ or periodic trajectories ${ }^{46}$ are found in some elegant analyses of photodissociative and other systems. One such example occurs with the predissociation of sodium iodide, where there is a simple quantitative semiclassical explanation of the fluctuations in linewidths with both $v$ and $j$, due to interference of two predissociation paths. ${ }^{74}$ Another is in the photodissociation of methyl iodide. ${ }^{73}$ While these latter problems have only been of the one- or two-coordinate type, the insight which occurred is significant. 
It will be interesting to see whether, stimulated by the current experimental excitement, a practical unifying approach to state-to-state chemical dynamics will be developed, into which the specific assumptions for the various classes of systems and models can be introduced, to permit the description of the wide range of behaviour that occurs in state-to-state systems. It is by no means assured that such a development will arise, and, indeed, largely numerical quantum methods are currently the ones predominating, as in the present Discussion, for treating real molecular systems.

From a practical point of view, should a method utilize classical trajectories in some manner, it would be useful for it to smooth over their frequent bifurcations. Examples have been found (e.g. ref. 75) where the quantum system is 'regular' (no 'overlapping avoided crossings' ${ }^{62 b, 76}$ ), while the corresponding classical system has chaotic regions! Similarly, even though that classical system was partly chaotic, accurate quantummechanical eigenvalues of the system could be obtained semiclassically by a perturbation theory based on an assumption that the classical motion was regular ('integrable'). ${ }^{77}$

Examples of treatments which in some sense do smooth over trajectories include those based on wavepackets ranging from simple (rigid Gaussians) to the computationally elaborate. ${ }^{78}$ Various limitations of these wavepacket calculations were recently summarized by Heller. ${ }^{78}$ A method that uses a Gaussian kernel in a Monte Carlo calculation of thermal properties, having as limits the quantum and stationary phase (semiclassical trajectory) values, has been reviewed recently. ${ }^{65}$ The future of theories for state-to-state dynamics continues to pose an interesting challenge, and the experimental results in the present Discussion provide examples of the broad range of data to be treated.

It is a pleasure to acknowledge the research support provided by the National Science Foundation. I have had helpful discussions with many colleagues, including John Simons, David Buckingham, Ahmed Zewail, Bill Miller and David Chandler.

This Discussion is one that Dick Bernstein would have enjoyed and participated in with his usual enthusiasm. He gave his time freely, and was a friend whose companionship, integrity, and youthful vitality we shall always remember.

\section{References}

1 J. O. Smith and H. S. Taylor, J. Chem. Phys., 1939, 7, 390.

2 E. W. R. Steacie, B de B. Darwent and W. R. Trost, Discuss. Faraday Soc, 1947, $2,80$.

3 R. A. Marcus, B. de B. Darwent and E. W. R. Steacie, J. Chem. Phys, 1948, 16, 987.

4 J. C. Polanyi, Faraday Discuss. Chem. Soc., 1979, 67, 129; B. A. Collings, J. C. Polanyi, M. A. Smith, A. Stolow and A. W. Tarr, Phys. Rev. Lett., 1987, 59, 2551; P. R. Brooks, R. F. Curl and T. C. Maguire, Ber. Bunsenges. Phys. Chem., 1982, 86, 401; P. R. Brooks, Chem. Rev., 1988, 88, 407.

5 E. Wigner, Trans. Faraday Soc., 1938, 34, 29.

6 E.g. E. Wigner, J. Chem. Phys., 1937, 5, 720; J. C. Keck, J. Chem. Phys., 1960, 32, 1035; J. C. Keck, Adv. Chem. Phys., 1967, 13, 85; R. L. Jaffe, J. M. Henry and J. B. Anderson, J. Chem. Phys., 1973, 59, 1128; J. B. Anderson, J. Chem. Phys. 1973, 58, 4684.

7 Cf. R. A. Marcus, J. Chem. Phys., 1966, 45, 2630.

8 M. Quack and J. Troe, Ber. Bunsenges. Phys. Chem., 1977, 81, 329; B. C. Garrett and D. G. Truhlar, J. Chem. Phys., 1979, 70, 1593; W. L. Hase, J. Chem. Phys., 1972, 57, 730; 1976, 64, 2442; D. G. Truhlar and B. C. Garrett, Annu. Rev. Phys. Chem., 1984, 35, 159.

9 R. A. Marcus, J. Phys. Chem., in the press.

10 A. H. Zewail, this Discussion.

11 A. Weaver and D. M. Neumark, this Discussion.

12 B. Soep, C. J. Whitham, A. Keller and J. P. Visticot, this Discussion.

13 E.g. M. J. Frost, P. Sharkey and I. W. M. Smith, this Discussion.

14 B. Katz, J. Park, S. Satyapal, S. Tasaki, A. Chattopadhyay, W. Yi and R. Bersohn, this Discussion.

15 C. A. Leach, A. A. Tsekouras, P. H. Vaccaro, R. N. Zare and D. Zhao, this Discussion.

16 J. J. Valentini, P. M. Aker, G. J. Germann and Y-D. Huh, this Discussion.

17 L. Schneider, K. Seekamp-Rahn, F. Liedeker, H. Steuwe and K. H. Welge, this Discussion. 
18 F. Green, G. Hancock and A. J. Orr-Ewing, this Discussion.

19 T. L. D. Collins, A. J. McCaffrey and M. J. Wynn, this Discussion.

20 B. Hayden and C. L. A. Lamont, this Discussion.

21 S. Holloway and X. Y. Chang, this Discussion.

22 K. J. Maynard, A. D. Johnson, S. P. Daley and S. T. Ceyer, this Discussion.

23 R. Schinke, K. Weide and B. Heumann, this Discussion.

24 M. Brouard, J. P. Simons and J-X. Wang, this Discussion.

25 M. H. Alexander, P. J. Dagdigian and H-J. Werner, this Discussion.

26 W. C. Stwalley, P. D. Kleiber, K. M. Sando, A. M. Lyyra, L. Li., S. Ananthamurthy, S. Bililign, H. Wang, J. Wang and V. Zafiropulos, this Discussion.

27 G. C. Schatz, D. Sokolovski and J. N. L. Connor, this Discussion.

$28 \mathrm{~K}$. Yamashita and K. Morokuma, this Discussion.

29 H. Metiu, this Discussion.

30 T. Seideman, J. L. Krause and M. Shapiro, this Discussion.

31 D. C. Chatfield, R. S. Friedman and D. G. Truhlar, this Discussion.

32 R. Kosloff and A. Hammerich, this Discussion.

33 R. A. Marcus, J. Chem. Phys., 1965, 43, 1598.

34 J. O. Hirschfelder and E. Wigner, J. Chem. Phys., 1939, 7, 616; M. A. Eliason and J. O. Hirschfelder, J. Chem. Phys., 1959, 30, 1426.

35 St. J. Dixon-Warren, E. T. Jensen, J. C. Polanyi, G-Q. Xu, S. H. Yang and H. C. Zeng, this Discussion.

36 C. E. D. Chidsey, Science, 1991, 251, 919.

37 P. Siddarth and R. A. Marcus, J. Phys. Chem., 1990, 94, 2985.

38 L. W. Peng, M. Dantus, A. H. Zewail, K. Kemnitz, J. M. Hicks and K. B. Eisenthal, J. Phys. Chem., $1987,91,6162$.

39 U. Leinhos, W. Kühnle and K. A. Zachariasse, J. Phys. Chem., 1991, 95, 2013.

40 D. G. Imre, J. L. Kinsey, A. Sinha and J. Krenos, J. Phys. Chem., 1984, 88, 3956; M. O. Hale, G. E. Galica, S. G. Glogover and J. L. Kinsey, J. Phys. Chem., 1986, 90, 4997.

41 E.g. S. Glasstone, k. J. Laidler and H. Eyring, The Theory of Rate Processes, McGraw-Hill, New York, 1941.

42 J. C. Polanyi, Discuss. Faraday Soc., 1967, 44, 293.

43 E.g. A. B. Elkowitz and R. E Wyatt, J. Chem. Phys., 1975, 62, 2504; 1975, 63, 702; F. Webster and J. C. Light, J. Chem. Phys., 1989, 90, 265; 1989, 90, 300.

44 E.g. J. Z. H. Zhang and W. H. Miller, Chem. Phys. Lett., 1987, 140, 329; J. Chem. Phys., 1988, 88, 4549; D. W. Schwenke, K. Haug, M. Zhao, D. G. Truhlar, Y. Sun, J. Z. H. Zhang and D. J. Kouri, J. Phys. Chem., 1988, 92, 3202; M. Mladenovic, M. Zhao, D. G. Truhlar, D. W. Schwenke, Y. Sun and D. J. Kouri, Chem. Phys. Lett., 1988, 146, 358; C. Yu, D. J. Kouri, M. Zhao, D. G. Truhlar and D. W. Schwenke, Chem. Phys. Lett., 1989, 157, 491.

45 R. A. Marcus, J. Chem. Phys., 1966, 45, 4493; 1986, 49, 2610.

46 W. H. Miller, N. C. Handy and J. E. Adams, J. Chem. Phys., 1980, 72, 99; M. V. Basilevski, Chem. Phys., 1977, 24, 81.

47 R. A. Marcus, Chem. Phys. Lett., 1988, 144, 208.

48 E. Pollak, Philos. Trans. R. Soc., Ser. A, 1990, 332, 343 and references cited therein; J. M. Gomez-Llorenti and H. S. Taylor, J. Chem. Phys., 1989, 91, 953; J. H. Gomez-Llorenti, J. Zakrzewski, H. S. Taylor and K. C. Kuhlander, J. Chem. Phys., 1989, 90, 1505, and references cited therein.

49 A. H. Zewail, this Discussion (Comment 153 ).

50 W. H. Green, A. J. Mahoney, Q-K. Zheng and C. B. Moore, J. Chem. Phys., 1991, 94, 1961; I-C. Chen, W. H. Green and C. B. Moore, J. Chem. Phys., 1988, 89, 314.

51 S. J. Klippenstein and R. A. Marcus, J. Chem. Phys., 1990, 93, 2418.

52 H. Sieber, E. Riedle and H. J. Neusser, J. Chem. Phys., 1988, 89, 4620; E. Riedle, H. J. Neusser and E. W. Schlag, J. Chem. Phys., 1981, 75, 4231.

53 A. Helman and R. A. Marcus, J. Chem. Phys., 1991, 95, 872.

54 W. R. S. Garton and F. S. Tomkins, Astrophys. J., 1969, 158, 839; A Holle, G. Wiebusch, J. Main, B. Hager, H. Rottke and K. H. Welge, Phys. Rev. Lett, 1986, 56, 2594; A. Holle, J. Main, G. Wiebusch, H. Rottke and K. H. Welge, Phys. Rev. Lett., 1988, 61, 161.

55 A. Carrington and R. A. Kennedy, J. Chem. Phys., 1984, 81, 91.

56 L. M. Delves, Nucl. Phys., 1958, 9, 391; 1960, 20, 275.

57 A. Kuppermann and G. C. Schatz, J. Chem. Phys., 1975, 62, 2502; Y. M. Wu, S. A. Cuccaro, P. G. Hipes and A. Kuppermann, Chem. Phys. Lett., 1990, 168, 429.

58 J. Manz and J. Römelt, Chem. Phys. Lett., 1981, 81, 179; Z. Bacic, J. D. Kress, G. A Parker and R. T. Pack, J. Chem. Phys., 1990, 92, 2344; G. C. Schatz, J. Chem. Phys., 1989, 90, 3582; J. Linderberg, S. B. Padkjaer, Y. Öhrn and B. Vessal, J. Chem. Phys., 1989, 90, 6254; J. M. Launay and M. LeDorneuf, Chem. Phys. Lett., 1989, 163, 178.

59 V. K. Babamov and R. A. Marcus, J. Chem. Phys., 1981, 74, 1790.

60 E. E. Nikitin and J. Troe, J. Chem. Phys., 1990, 92, 6594; E. I. Dashevskaya, E. E. Nikitin and J. Troe, J. Chem., Phys., 1990, 93, 7803. 
61 E.g. M. C. Gutzwiller, Chaos in Classical and Quantum Mechanics, Springer, New York, 1990.

62 (a) E.g. W. Eastes and R. A. Marcus, J. Chem. Phys., 1974, 61, 4301; D. W. Noid and R. A. Marcus, J. Chem. Phys., 1975, 62, 2119; I. C. Percival, Adv. Chem. Phys., 1977, 36, 1; N. C. Handy, in ref. 70, below, p. 297; (b) M. V. Berry, Ann. Phys., 1981, 131, 163; D. W. Noid, M. L. Koszykowski and R. A. Marcus, Annu. Rev. Phys. Chem., 1981, 32, 267.

63 G. A. Voth, D. Chandler and W. H. Miller, J. Chem. Phys., 1989, 91, 7749; M. J. Gillan, J. Phys. C, 1987, 20, 3621; G. A. Voth, J. Chem. Phys., 1991, 94, 4095; Chem. Phys. Lett., 1990, 170, 289; A. C. Stuchebrukhov, J. Chem. Phys., in the press.

64 E.g. B. J. Berne and D. Thirumalai, Annu. Rev. Phys. Chem., 1986, 37, 401.

65 J. D. Doll, D. L. Freeman and T. L. Beck, Adv. Chem. Phys., 1990, 78, 61; N. Makri, Comput. Phys. Commun., 1991, 63, 389.

66 W. H. Miller, S. D. Schwartz and J. W. Tromp, J. Chem. Phys., 1983, 79, 4889; J. D. Doll, J. Chem. Phys., 1984, 81, 3536.

67 M. C. Gutzwiller, J. Math. Phys., 1967, 8, 1979; 1971, 12, 343; P. Pechukas, Phys. Rev., 1969, 181, 165, 174.

68 W. H. Miller, Adv. Chem. Phys., 1974, 25, 69; 1975, 30, 77.

69 R. A. Marcus, J. Chem. Phys., 1972, 56, 311.

70 M. S. Child, Semiclassical Methods in Molecular Scattering and Spectroscopy, D. Reidel, Dordrecht, 1979.

71 E.g. H. Kreek, R. L. Ellis and R. A. Marcus, J. Chem. Phys., 1975, 62, 913.

72 H. Kreek and R. A. Marcus, J. Chem. Phys., 1974, 61, 3308.

73 E. E. Nikitin and M. Ya. Ovchinnikova, Chem. Phys., 1989, 138, 45.

74 S. Chapman and M. S. Child, J. Phys. Chem., 1991, 95, 578; S. H. Schaefer, D. Bender and E. Tiemann, Chem. Phys., 1984, 89, 65.

75 D. W. Noid, M. L. Koszykowski, M. Tabor and R. A. Marcus, J. Chem. Phys., 1980, $72,6169$.

76 R. A. Marcus, Faraday Discuss. Chem. Soc., 1983, 75, 103; in Horizons of Quantum Chemistry, Proc. Third Int. Congress Quantum Chem., Kyoto, 1979, ed. K. Fukui and B. Pullman, D. Reidel, Dordrecht, 1980, p. 107.

77 R. T. Swimm and J. B. Delos, J. Chem. Phys., 1979, 71, 1706; C. Jaffé and W. P. Reinhardt, J. Chem. Phys., 1979, 71, 1862.

78 E. J. Heller, J. Chem. Phys., 1991, 94, 2723.

Received 29th July, 1991 